Relations industrielles

Industrial Relations

\title{
Les métiers qui meurent dans l'industrie du bâtiment
}

\section{Marcel Clément}

Volume 4, numéro 10, juin 1949

URI : https://id.erudit.org/iderudit/1023545ar

DOI : https://doi.org/10.7202/1023545ar

Aller au sommaire du numéro

Éditeur(s)

Département des relations industrielles de l'Université Laval

ISSN

0034-379X (imprimé)

1703-8138 (numérique)

Découvrir la revue

Citer cet article

Clément, M. (1949). Les métiers qui meurent dans l'industrie du bâtiment. Relations industrielles / Industrial Relations, 4(10), 94-97.

https://doi.org/10.7202/1023545ar

Tous droits réservés (C Département des relations industrielles de l’Université Laval, 1949
Ce document est protégé par la loi sur le droit d'auteur. L'utilisation des services d'Érudit (y compris la reproduction) est assujettie à sa politique d'utilisation que vous pouvez consulter en ligne.

https://apropos.erudit.org/fr/usagers/politique-dutilisation/ 
encore embauché, on ne fait que l'empêcher d'obtenir un emploi et rien n'empêche la clause d'avoir son effet, mais s'il est déjà au service de l'entreprise, l'union ne peut alors exiger le renvoi.

La loi fédérale a donc voulu permettre ex- pressément les clauses de sécurité syndicale tout en sauvegardant le droit des salariés de quitter une union jouissant de l'atelier fermé, pour adhérer à une autre association destinée à la supplanter comme agence de négociation collective.

\section{LES MÉTIERS QUI MEURENT DANS L'INDUSTRIE DU BÂTIMENT}

\section{MARCEL CléMENT}

Au cours de l'enquête que nous avons poursuivie sur la situation de la main-d'oeuvre dans l'industrie du bâtiment de la province de Québec, nous avons été amenés à enregistrer deux phénomènes particulièrement nets, et dont les conséquences, à longue échéance, risquent, si l'on n'y porte un prompt remède, d'être fort onéreuses. D'une part, il est apparu que certaines catégories de métiers jouissaient parmi les jeunes, d'un succès croissant et incontrôlé, et d'autre part, certaines autres catégories sont désertées à un rythme inquiétant.

Le tableau I ci-dessous indique l'effectif, par groupe d'âge, des apprenitis et des salariés qualifiés des huit principaux métiers de l'Industrie du bâtiment, tel qu'il a été révélé par un questionnaire rempli à la date du 31 juillet 1948. On tiendra compte de ce que ces chiffres:

a) ne recouvrent ni les contracteurs, ni les artisans installés à leur compte, ni les journaliers, mais exclusivement les salariés qualifiés des métiers intéressés;

b) ne concernent que les régions contrôlées par un comité paritaire.

TABLEAU 1: EFFECTIF ETABLI PAR GROUPE D'AGE, DES APPRENTIS ET DES SALARIES QUALIFIES DES HUIT PRINCIPAUX METIERS DE L'INDUSTRIE DU BATIMENT DANS LA PROVINCE DE QUEBEC, AU 31 JUILLET 1949

\begin{tabular}{|c|c|c|c|c|c|c|c|}
\hline $\begin{array}{l}\text { Nomenclature } \\
\text { des } \\
\text { métiers }\end{array}$ & $\begin{array}{l}\text { Effectif } \\
\text { des } \\
\text { apprentis }\end{array}$ & $\begin{array}{l}\text { Salariés } \\
\text { ayant } \\
\text { moins de } \\
25 \text { ans }\end{array}$ & $\begin{array}{c}\text { Salariés } \\
\text { ayant } \\
\text { de } 25 \text { à } \\
35 \text { ans }\end{array}$ & $\begin{array}{c}\text { Salariés } \\
\text { ayant } \\
\text { de } 35 \text { à } \\
45 \text { ans }\end{array}$ & $\begin{array}{c}\text { Salariés } \\
\text { ayant } \\
\text { de } 45 \text { à } \\
55 \text { ans }\end{array}$ & $\begin{array}{c}\text { Salariés } \\
\text { ayant } \\
\text { plus de } \\
55 \text { ans }\end{array}$ & $\begin{array}{c}\text { Total des } \\
\text { salariés } \\
\text { employés } \\
\text { dans chaque } \\
\text { métier } \\
\end{array}$ \\
\hline Charpentiers-menuisiers & 1,529 & 795 & 2,561 & 2,711 & 1,919 & 1,231 & 10,746 \\
\hline Briqueteurs & 367 & 199 & 477 & 452 & 517 & 242 & 2,254 \\
\hline Plâtriers-maçons & 308 & 74 & 201 & 381 & 320 & 158 & 1,442 \\
\hline Peintres & 706 & 432 & 1,156 & 1,522 & 818 & 336 & 4,970 \\
\hline Plombiers & 2,321 & 487 & 780 & 727 & 326 & 187 & 4,828 \\
\hline $\begin{array}{l}\text { Poseurs d'appareils } \\
\text { de chauffage }\end{array}$ & 378 & 82 & 134 & 95 & 54 & 20 & 763 \\
\hline Ferblantiers & 271 & 59 & 227 & 193 & 132 & 65 & 947 \\
\hline Electriciens & 1,557 & 614 & 795 & 472 & 242 & 35 & 3,715 \\
\hline $\begin{array}{l}\text { Total des salariés établi } \\
\text { par groupe d’âge }\end{array}$ & 7,437 & 2,742 & 6,331 & 6,553 & 4,328 & 2,274 & 29,665 \\
\hline
\end{tabular}


TABLEAU 2: ANALYSE DES STATISTIQUES DU TABLEAU 1

\begin{tabular}{l|c|c}
\hline \multicolumn{1}{c|}{$\begin{array}{c}\text { Nomenclature } \\
\text { des métiers }\end{array}$} & $\begin{array}{c}\text { Age moyen } \\
\text { des salariés } \\
\text { des chaque } \\
\text { métier }\end{array}$ & $\begin{array}{c}\text { \% des apprentis } \\
\text { de chaque metier } \\
\text { par rapport a } \\
\text { peffectuf total } \\
\text { des salartes } \\
\text { du mettier }\end{array}$ \\
\hline $\begin{array}{l}\text { Charpentiers- } \\
\text { menuisiers }\end{array}$ & 40 ans & $14.22 \%$ \\
\hline Briqueteurs & 41 ans & $16.28 \%$ \\
\hline $\begin{array}{l}\text { Plâtriers- } \\
\text { maçons }\end{array}$ & 43 ans & $21.35 \%$ \\
\hline $\begin{array}{l}\text { Peintres } \\
\text { Plombiers }\end{array}$ & 39 ans & $14.20 \%$ \\
\hline $\begin{array}{l}\text { Poseurs d'appareils } \\
\text { de chauffage }\end{array}$ & 35 ans & $49.54 \%$ \\
\hline $\begin{array}{l}\text { Ferblantiers } \\
\text { Electriciens }\end{array}$ & 39 ans & $28.61 \%$ \\
\hline
\end{tabular}

On observera clairement les tendances qui se dégagent de ces chiffres si l'on considère attentivement le tableau II, où l'on a rapproché, dans chaque métier, l'âge moyen des salariés et le pourcentage des apprentis (en comparaison de l'effectif total des salariés du métier). On constatera que plus l'âge moyen est bas, c'est-à-dire plus le nombre des jeunes est considérable dans le mé- tier, et plus le pourcentage des apprentis est élevé, ce qui prouve que l'on a affaire à une tendance qui remonte au moins à une dizaine d'année, et qui persiste en s'aggravant. Au contraire, plus l'âge moyen est élevé, plus le pourcentage des apprentis est faible, ce qui montre que, inversement, l'abandon de ces métiers est déjà ancien et luimême menaçant pour l'avenir.

Dans l'ensemble, on peut résumer la situation en distinguant deux tendances fondamentales.

a) Une tendance régressive des métiers traditionnels: charpente, menuiserie, briquetage, maçonnerie, peinture. Cette tendance est particulièrement grave dans la charpente et dans la peinture; elle est très sérieuse dans le briquetage et la maçonnerie. A s'en tenir à la cadence actuelle, et compte tenu de la moyenne d'âge élevée dans ces métiers, d'ici quinze ans, la moitié de l'effectif actuel sera relayé, non par des apprentis formés, mais par des manoeuvres titulaires d'une carte de compétence.

b) Une tendance progressive des métiers mécaniques: plombiers, ferblantiers, poseurs d'appareils de chauffage, électriciens. Le développement de l'apprentissage semble être une véritable «mode» dans les métiers placés sous le contrôle du gouvernement. Il est significatif à cet égard de constater que l'effectif des seuls apprentis plombiers et électriciens s'élève à 3,878 , soit à plus de $50 \%$ de l'effectif total de l'apprentissage dans les huit métiers étudiés.

On se fera une idée frappante du phénomène si l'on compare les deux graphiques suivants, dont l'un illustre la structure actuelle des métiers du bâtiment, l'autre la structure probable, sur la base des tendances indiquées, d'ici une vingtaine d'années.

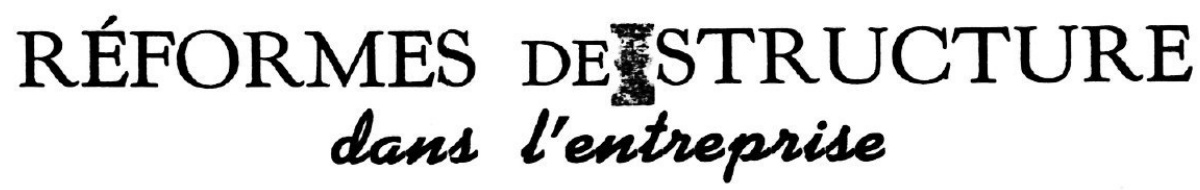

Un livre de 210 pages de P.-E. Bolté, Marcei Clément et Gérard Dion

En vente au prix de $\$ \mathbf{1 . 0 0}$

au Département des relations industrielles, 2, rue de l'Université, Québec. 


\section{Schéma I}

Structure actuelle du rapport existant entre les effectifs de huit métiers

du bâtiment.

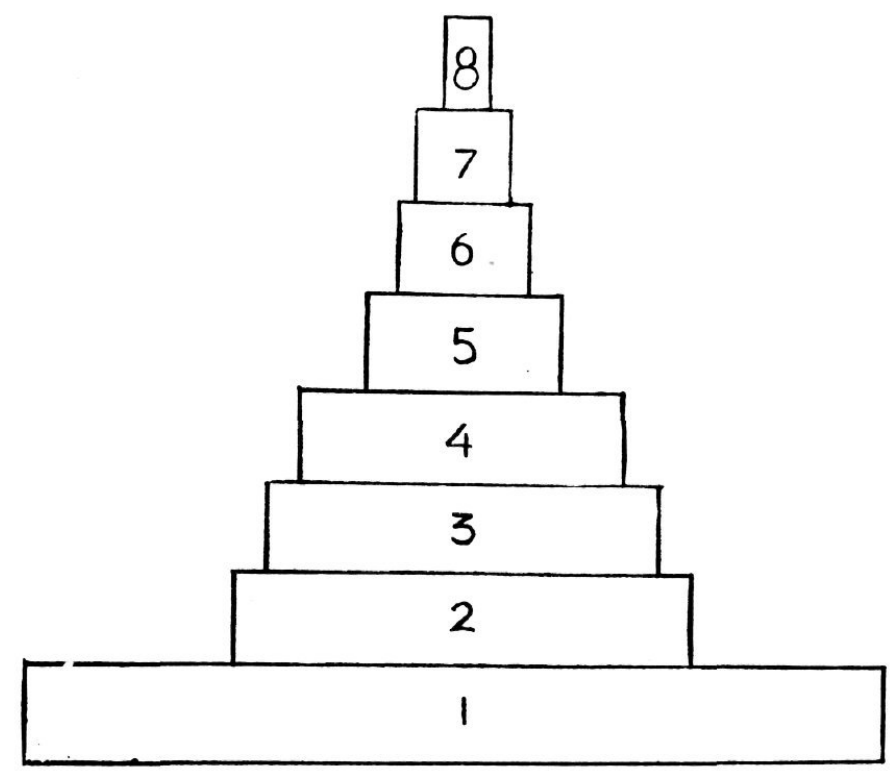

Schéma II

Structure, en 1970, du rapport existant entre les effectifs de huit métiers

du batiment.

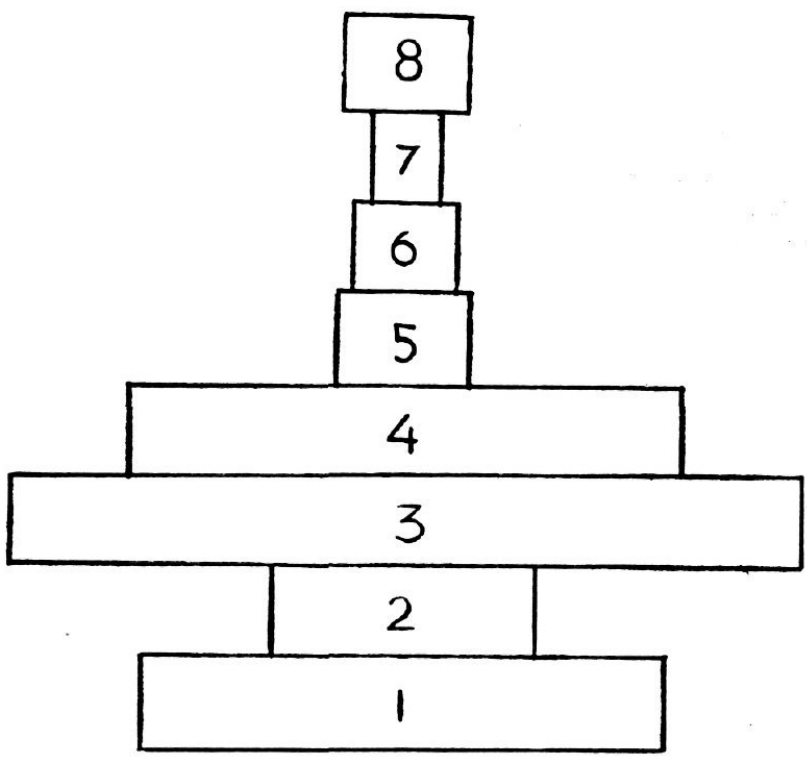

1: Charpentiers-menuisiers - 2: Peintres - 3: Plombiers - 4: Electriciens - 5: Briqueteurs 6: Plâtriers - 7 : Ferblantiers - 8: Poseurs d'appareils de chauffage

II n'est pas douteux que, par leur seule nature, certains metiers attirent les jeunes et que certains autres les rebutent. A supposer que ce soit là la cause principale des variations du pourcentage des apprentis, on aurait, suivant les métiers, un ordre de préférence décroissant:

\begin{tabular}{c|lr} 
Ordre & Métiers & \% des app \\
\hline 1 & Poseurs d'ap. de chauffage & 4.9 \\
2 & Plombiers & 4.8 \\
3 & Electriciens & 4.1 \\
4 & Ferblantiers & 2.8 \\
5 & Plâtriers-maçons & 2.1 \\
6 & Briqueteurs & 1.6 \\
7 & Peintres & 1.4 \\
8 & Charpentiers & 1.4
\end{tabular}

Il est clair que les quatre métiers les plus recherchés sont:

- des métiers mécaniques;

- des métiers requérant nécessairement un apprentissage;
- des métiers de forte technicité;

- des métiers - pour les plus importants - contrôlés par l'Etat;

- des métiers où la réparation est aussi fréquente que l'ouvrage neuf;

- des métiers s'exerçant plus généralement à l'intérieur qu'à l'extérieur.

Il est clair, au contraire, que les métiers moins recherchés sont:

- des métiers traditionnels;

- des métiers auxquels participent généralement des journaliers sans apprentissage;

- des métiers que lon croit de faible technicité, en raison de la connaissance largement répandue des opérations les plus élémentaires (clouer, scier, peindre);

- des métiers dont le contrôle est assuré, à Montréal, par les Syndicats, et ailleurs, par les Comités paritaires;

- des métiers où (en particulier dans les nos 5 et 6) la réparation est moins fréquente que l'ouvrage neuf, et donc plus soumis aux cycles économiques;

- des métiers qui s'exercent exclusivement ou principalement à l'extérieur, et dont le caractère saisonnier est par conséquent très accentué. 
Il en résulte:

- un problème de sélection professionnelle: Les jeunes, les plus doués, se dirigent vers les métiers mécaniques. Le recrutement des métiers traditionnels est donc insuffisant en quantité, mais aussi en qualité;

- un problème social: Il en résulte une grave chute de compétence dans les métiers traditionnels qui se composent de plus en plus d'anciens journaliers et des candidats qui ne se sont pas sentis capables de passer l'examen des métiers mécaniques.

D’où un déclin rapide de la compétence qui entraîne une «mauvaise conscience » chez les jeunes couches de ces professions qui ne se sentent pas «capables»;

- un problème économique: L'augmentation des prix de revient de la main-d'oeuvre dans les métiers traditionnels et l'éventualité d'un chômage futur dans les métiers mécaniques.

c) Parmi les entrepreneurs et les compagnons, les tendances actuelles vont dans le même sens que parmi les jeunes. Les entrepreneurs et compagnons des métiers mécaniques acceptent facilement le quota $1 / 1$. Les entrepreneurs et compa- gnons des métiers traditionnels s'attachent aussi étroitement que possible au quota 1/5. D'où il résulte:

- un problème social: l'attitude des syndicats;

- un problème économique: l'attitude des entrepreneurs.

La prise de conscience de ces faits constitue la démarche initiale de tout effort de redressement. Il n'est pas douteux que l'augmentation des effectifs des métiers mécaniques ne corresponde à une nécessité. Mais le rythme de cette augmentation ne peut-il constituer, dès maintenant, un danger ? Il appartient à la profession de l'examiner. Et, au rebours, l'abandon des métiers traditionnels, doit être, d'urgence, contenu. L'effort des centres de préapprentissage représente une mesure de grande portée économique et sans doute la solution la plus efficace au problème. Aussi doit-on souhaiter que leur nombre s'accroisse et que, de plus en plus, la préférence soit accordée aux jeunes assez consciencieux pour accepter de recevoir, pendant une période de six mois à un an, une formation qui oriente toute la vie, car elle leur donne ce qui fait le plus défaut: la joie au travail, privilège de la compétence.

\section{L'ÉCONOMIE DOIT ÊTRE PLACÉE AU SERVICE DE L'HOMME *}

Paul-H. Flairondon

L'importance de ces entretiens apparaît dans une vive lumière si l'on s'arrête à méditer sur le thème qui en fait l'objet. Vous vous êtes réunis, messieurs, dans l'intention d'examiner les conditions d'une économie mondiale libre, au service du consommateur, dans un monde pacifique. Le choix d'un tel programme est un témoignage de haute portée. Il établit en mème temps votre souci de réalisme économique, votre désir de promotion morale de la personne humaine et enfin votre volonté tout à la fois lucide et confiante dans le destin d'un monde enfin réconcilié dans la vraie paix.

Messieurs, vous savez sans doute combien ce continent nord-américain demeure attaché à une liberté qui n'a jamais cessé de fournir un cadre suffisamment souple à l'essor de production le plus considérable que l'histoire ait connu. Liberté d'en-

\footnotetext{
* Nous reproduisons ici le texte de l'allocution prononcée, le 15 juin 1949. par M. Paul-H. Plamondon, président de la Chambre de Commerce de Québec au congrès de la Chambre de Commerce internationale, Québec.
}

treprise, dont les expériences totalitaires nous font mieux comprendre la fécondité en vue du bien commun et la valeur d'épanouissement personnei. N'est-ce pas, en effet, un défi à la nature même de l'homme, que de limiter le ròle du chef d'entreprise aux limites disciplinaires d'un dirigisme d'Etat qui finit toujours par briser les ressorts intimes de l'esprit d'entreprise, de l'esprit de risque et par décourager les lutteurs les mieux armés pour créer de la prospérité ?

A l'heure où la technique moderne rend l'univers de plus en plus conscient de son unité, où le progrès des travaux scientifiques met à notre portée une information toujours plus précise et plus riche, comment imaginer le retour à un nationalisme économique fermé, dont l'égoïsme, d'ailleurs mal compris, se retourne régulièrement contre soimême. Toutefois, messieurs, l'heure est trop grave pour que nous vous dissimulions certaines vérités. L'économie n'a pas toujours été au service du consommateur et celui-ci, parfois, n’a été considéré que comme un instrument ou un moyen; non com- 